\title{
Respiratory Failure Related to Central Nervous System Disorder
}

National Cancer Institute

\section{Source}

National Cancer Institute. Respiratory Failure Related to Central Nervous System

Disorder. NCI Thesaurus. Code C101332.

Impaired gas exchange by the respiratory system resulting in hypoxemia and decreased

oxygenation of the tissues that may be associated with increased arterial levels of carbon dioxide; the respiratory failure is due to a central nervous system disorder. 